\title{
What is the optimal pharmacological management of retained placenta?
}

In this Practice article (BMJ 2014;349:g4778, doi:10.1136/bmj. g4778) by Duffy and colleagues, an error occurred in the first paragraph. The authors provided a correction [http://www.bmj. com/content/349/bmj.g5173] which requires further clarification.

The second sentence should now read: "The third stage of labour can be managed actively or physiologically, defined by the National Institute for Health and Care Excellence (NICE) ${ }^{1}$ as follows: Physiological management of the third stage involves a package of care which includes all of these three components: no routine use of uterotonic drugs, no clamping of the cord until pulsation has ceased, and delivery of the placenta by maternal effort.

Active management of the third stage involves a package of care which includes all of these three components: routine use of uterotonic drugs, early clamping and cutting of the cord, and controlled cord traction."

National Institute for Health and Care Excellence. Intrapartum care. CG55. 2007. www. nice.org.uk/guidance/cg55.

Cite this as: BMJ 2014;349:96476

๑ B BMJ Publishing Group Ltd 2014 